\title{
Cross frequency coupling in next generation inhibitory neural mass models
}

\author{
Andrea Ceni, ${ }^{1}$ Simona Olmi, ${ }^{2,3}$ Alessandro Torcini, ${ }^{4,3}$ and David Angulo-Garcia ${ }^{5}$ \\ ${ }^{1)}$ Department of Computer Science, College of Engineering, Mathematics and Physical Sciences, University of Exeter, \\ Exeter EX4 $4 Q F, U K$ \\ ${ }^{2)}$ Inria Sophia Antipolis Méditerranée Research Centre, 2004 Route des Lucioles, 06902 Valbonne, \\ France \\ ${ }^{3)}$ CNR - Consiglio Nazionale delle Ricerche - Istituto dei Sistemi Complessi, via Madonna del Piano 10, \\ 50019 Sesto Fiorentino, Italy \\ 4) Laboratoire de Physique Théorique et Modélisation, Université de Cergy-Pontoise, CNRS, UMR 8089, \\ 95302 Cergy-Pontoise cedex, France \\ ${ }^{5)}$ Grupo de Modelado Computacional - Dinámica y Complejidad de Sistemas. Instituto de \\ Matemáticas Aplicadas. Carrera 6 \#36 - 100. Universidad de Cartagena. Cartagena de Indias, \\ Colombia
}

Coupling among neural rhythms is one of the most important mechanisms at the basis of cognitive processes in the brain. In this study we consider a neural mass model, rigorously obtained from the microscopic dynamics of an inhibitory spiking network with exponential synapses, able to autonomously generate collective oscillations (COs). These oscillations emerge via a super-critical Hopf bifurcation, and their frequencies are controlled by the synaptic time scale, the synaptic coupling and the excitability of the neural population. Furthermore, we show that two inhibitory populations in a master-slave configuration with different synaptic time scales can display various collective dynamical regimes: namely, damped oscillations towards a stable focus, periodic and quasi-periodic oscillations, and chaos. Finally, when bidirectionally coupled the two inhibitory populations can exhibit different types of $\theta-\gamma$ cross-frequency couplings (CFCs): namely, phase-phase and phase-amplitude CFC. The coupling between $\theta$ and $\gamma$ COs is enhanced in presence of a external $\theta$ forcing, reminiscent of the type of modulation induced in Hippocampal and Cortex circuits via optogenetic drive.

In healthy conditions, the brain's activity reveals a series of intermingled oscillations, generated by large ensembles of neurons, which provide a functional substrate for information processing. How single neuron properties influence neuronal population dynamics is an unsolved question, whose solution could help in the understanding of the emergent collective behaviors arising during cognitive processes. Here we consider a neural mass model, which reproduces exactly the macroscopic activity of a network of spiking neurons. This mean-field model is employed to shade some light on an important and ubiquitous neural mechanism underlying information processing in the brain: the $\theta-\gamma$ cross-frequency coupling. In particular, we will explore in detail the conditions under which two coupled inhibitory neural populations can generate these functionally relevant coupled rhythms.

\section{INTRODUCTION}

Neural rhythms are the backbone of information coding in the brain ${ }^{13}$. These rhythms are the proxy of large populations of neurons that orchestrate their activity in a regular fashion and selectively communicate with other populations producing complex functional interactions $^{15,52}$.

One of the most prominent rhythmic interaction appearing in the brain is the so-called $\theta-\gamma$ coupling, exhibited between the slow oscillating $\theta$ band $(4 \mathrm{~Hz}-12 \mathrm{~Hz})$ and the faster $\gamma$ rhythm $(25-100 \mathrm{~Hz})^{34}$. This specific frequency interaction is an example of a more general mechanism termed Cross-Frequency-Coupling $(\mathrm{CFC})^{15,28}$. CFC has been proposed to be at the basis of sequence representation, long distance communication, sensory parsing and de-multiplexing ${ }^{25,28}$. CFC can manifest in a variety of ways depending on the type of modulation that one rhythm imposes on the other (Amplitude-Amplitude, Amplitude-Frequency, PhaseAmplitude, Frequency-Frequency, Frequency-Phase or Phase-Phase) ${ }^{28}$.

In this article, we will focus on Phase-Phase (P-P) and Phase-Amplitude (P-A) couplings of $\theta$ and $\gamma$ rhythms. In particular, $\mathrm{P}-\mathrm{P}$ coupling refers to n:m phase locking between gamma and theta phase oscillations ${ }^{46}$ and it has been demonstrated to play a role in visual tasks in humans ${ }^{24}$ and it has been identified in the rodent hippocampus during maze exploration ${ }^{5}$. The P-A coupling (or $\theta$-nested $\gamma$ oscillations) corresponds to the fact that the phase of the theta-oscillation modifies the amplitude of the gamma waves and it has been shown to support the formation of new episodic memories in the human hippocampus ${ }^{33}$ and to emerge in various part of the rodent brain during optogenetic theta stimulations in vitro $^{2,10,11,42}$.

In an attempt to understand the complex interactions emerging during CFC, several mathematical models describing the activity of a large ensemble of neurons have been proposed ${ }^{19}$. Widely known examples include the phenomenologically based Wilson-Cowan ${ }^{55}$ and Jansen$\mathrm{Rit}^{27}$ neural mass models. While the usefulness of these heuristic models is out of any doubt, they are not related to any underlying microscopic dynamics of a neuronal 
population. A second approach consists on the use of a mean-field (MF) description of the activity of a large ensemble of interacting neurons ${ }^{8,9,16,39,40,45,49}$. Although in this latter MF framework some knowledge is gained on the underlying microscopic dynamics that manifests at the macroscopic level, it comes with the downside of several, not always verified, assumptions about the statistics of single neuron firings ${ }^{50}$.

Recently, it has been possible to obtain in an exact manner a macroscopic description of an infinite ensemble of pulse-coupled Quadratic Integrate-and-Fire (QIF) neurons ${ }^{18,36,38,43}$. This result has been achieved thanks to the Ott-Antonsen ansatz, which allows to exactly obtain the macroscopic evolution of phase oscillator networks fully coupled via purely sinusoidal field ${ }^{41}$. In particular, in ${ }^{38}$ the authors have been able to derive for QIF neurons with instantaneous synapses an exact neural mass model in terms of the firing rate and the average membrane potential. This exactly reduced model allows for the first time to understand the effects of the microscopic neural dynamics at the macroscopic level without making use of deliberate assumptions on the firing statistics.

In this paper, we employ the exactly reduced model introduced in ${ }^{20}$ for QIF neurons with exponential synapses to study the emergence of mixed-mode oscillations in inhibitory networks with fast and slow synaptic kinetics. In particular, in sub-section III A we will characterize the dynamical behavior of a single population and review the conditions required to observe collective oscillations (COs). Afterwards, in sub-section III B, we will analyze the response of the population to a modulating signal paying special attention to the emergence of phase synchronization. We will then consider the possible macroscopic dynamics displayed by two coupled populations characterized by different synaptic time scales in a master-slave configuration (sub-section III C) and in a bidirectional set-up (sub-section III D). In this latter case we will focus on the conditions for the emergence of $\theta-\gamma$ CFCs among the COs displayed by the two populations.

\section{MODEL AND METHODS}

\section{A. Network Dynamics}

Through this paper we will consider either one or two populations of QIF neurons interacting via inhibitory post-synaptic potentials (IPSPs) with an exponential profile. In this framework, the activity of the population $l \in\{A, B\}$ is described by the dynamics of the membrane potentials $V_{i}^{(l)}$ of its neurons and of the associated synaptic fields $S_{i}^{(l)}$. Here we will assume a fully coupled topology for all networks, hence each neuron within a certain population is subject to the same synaptic field $S^{(l)}$, where the neuron index has been dropped. Therefore, the dynamics of the network can be written as

$$
\begin{aligned}
\tau \dot{V}_{i}^{(l)} & =\left(V_{i}^{(l)}\right)^{2}+\eta_{i}^{(l)}+J_{l l} \tau S^{(l)}+J_{k l} \tau S^{(k)}+I^{(l)}(t) \\
\tau_{d}^{(l)} \dot{S}^{(l)} & =-S^{(l)}+\frac{1}{N^{(l)}} \sum_{t_{j}^{(l)}} \delta\left(t-t_{j}^{(l)}\right) \\
i & =1, \ldots, N^{(l)} \quad l, k \in\{A, B\}
\end{aligned}
$$

where $\tau=10 \mathrm{~ms}$ is the membrane time constant which is assumed equal for both populations, $\eta_{i}^{(l)}$ is the excitability of the $i^{t h}$ neuron of population $l, J_{l k}$ is the strength of the inhibitory synaptic coupling of population $l$ acting on population $k$ and $I^{(l)}(t)$ is a time dependent external current applied on population $l$. The synaptic field $S^{(l)}(t)$ is the linear super-position of the all exponential IPSPs $s(t)=\mathrm{e}^{-t / \tau_{d}^{(l)}}$ emitted within the $l$ population in the past. Due to the quadratic term in the membrane potential evolution, which allows the variable to reach infinity in a finite time, the emission of the $j^{\text {th }}$ spike in the network occurs at time $t_{j}^{(l)}$ whenever $V_{i}^{(l)}\left(t_{j}^{(l)_{-}}\right) \rightarrow+\infty$, while the reset mechanism is modeled by setting $V_{i}^{(l)}\left(t_{j}^{(l)_{+}}\right) \rightarrow-\infty$, immediately after the spike emission.

For reasons that will be clear in the next paragraph we will also assume that the neuron excitability values $\eta_{i}^{(l)}$ are randomly distributed according to a Lorentzian probability density function (PDF)

$$
g_{l}(\eta)=\frac{1}{\pi} \frac{\Delta^{(l)}}{\left(\eta-\bar{\eta}^{(l)}\right)^{2}+\left(\Delta^{(l)}\right)^{2}},
$$

where $\bar{\eta}^{(l)}$ is the median and $\Delta^{(l)}$ is the half-width halfmaximum (HWHM) of the PDF. For simplicity, we set $\bar{\eta}=1$ throughout the paper, unless otherwise stated.

In order to characterize the macroscopic dynamics we will employ the following indicators:

$r^{(l)}(t)=\frac{1}{N^{(l)} \Delta t} \sum_{t_{j}^{(l)}} \delta\left(t-t_{j}^{(l)}\right), \quad v^{(l)}(t)=\frac{1}{N^{(l)}} \sum_{j}^{N^{(l)}} V_{j}^{(l)}(t)$,

which represent the average population activity of each network and the average membrane potential, respectively. In particular the average population activity of the $l$-network $r^{(l)}(t)$ is given by the number of spikes emitted in a time unit $\Delta t$, divided by the total number of neurons. Furthermore, the emergence of COs in the dynamical evolution, correspondng to periodic motions of $r^{(l)}(t)$ and $v^{(l)}(t)$, will be characterized in terms of their frequencies $\nu^{(l)}$.

\section{B. Mean-Field Evolution:}

In the limit of infinitely many neurons $N^{(l)} \rightarrow \infty$ we can derive, for each population $l$, the evolution of the 

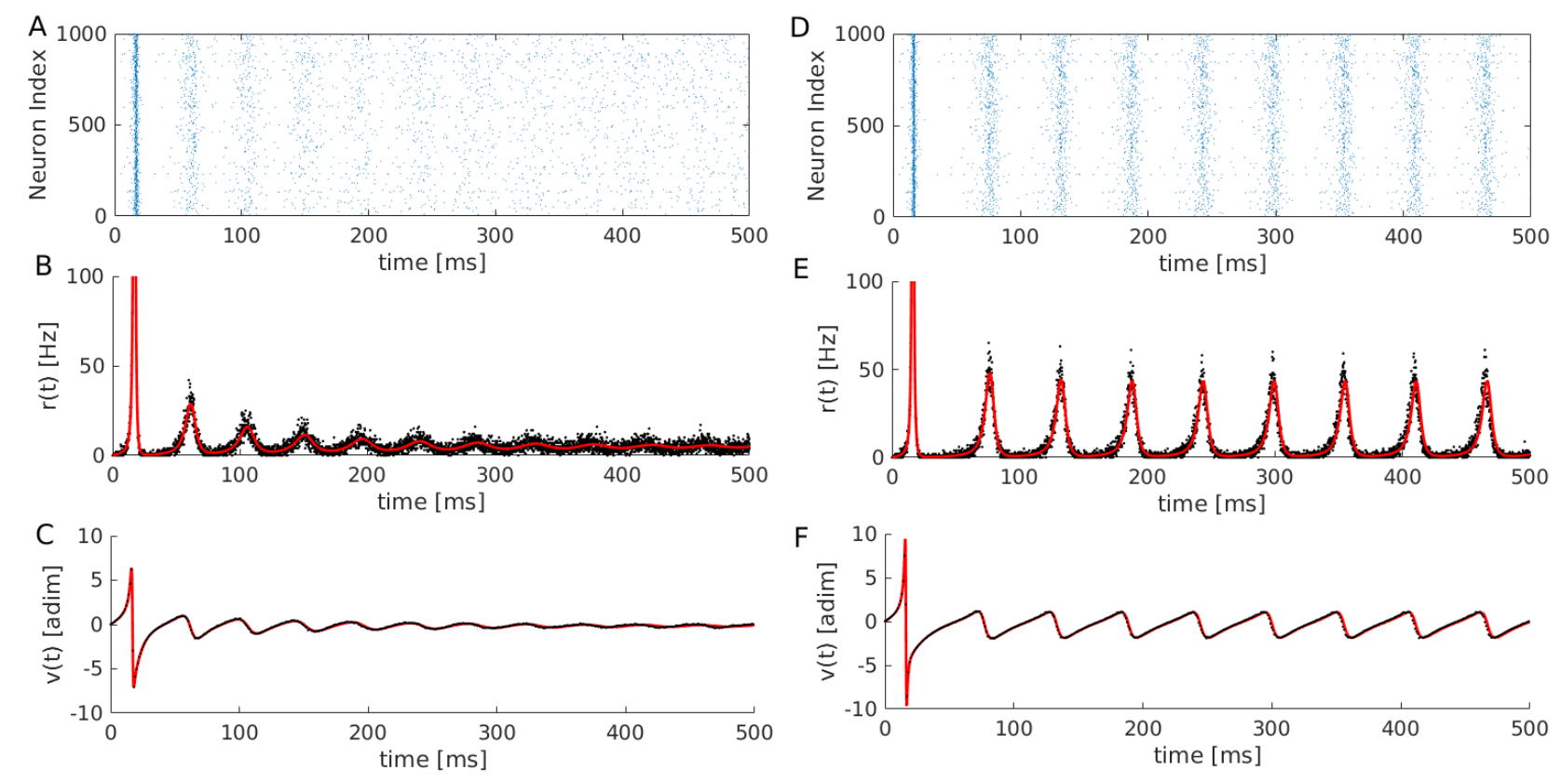

FIG. 1. Oscillations in a self inhibited QIF population: Left (right) column refers to damped (self-sustained) COs. From top to bottom: raster plots (A)-(D); istantaneous firing rate $r(t)(\mathrm{B})-(\mathrm{E})$; average membrane potential $v(t)(\mathrm{C})-(\mathrm{F})$. The network simulations are reported as black dots, while the MF dynamics (5) is shown as a red line. For the network simulation $N=10000$ QIF units are simulated. Left (right) panels were obtained with $\tau_{d}=3 \mathrm{~ms}\left(\tau_{d}=8 \mathrm{~ms}\right)$. Other parameters $\bar{\eta}=1, \Delta=0.05$, $J=-20$ and $\tau=10 \mathrm{~ms}$.

$\operatorname{PDF} \rho^{(l)}(V \mid \eta, t)$ describing the probability distribution of finding a neuron with potential $V$ and excitability $\eta$ at a time $t$ via the continuity equation:

$$
\begin{aligned}
\frac{\partial \rho^{(l)}(V \mid \eta, t)}{\partial t} & =-\frac{\partial \mathcal{F}^{(l)}(V \mid \eta, t)}{\partial V^{(l)}} \\
\mathcal{F}^{(l)}(V \mid \eta, t) & =\rho^{(l)}\left(\left(V^{(l)}\right)^{2}+\eta^{(l)}+\mathcal{J}^{(l)}\right),
\end{aligned}
$$

where $\mathcal{F}^{(l)}(V \mid \eta, t)$ is the probability flux while $\mathcal{J}^{(l)}=$ $J_{l l} \tau S^{(l)}+J_{k l} \tau S^{(k)}+I^{(l)}(t)($ for $l, k \in\{A, B\})$ is the contribution of all synaptic currents plus the external input. The continuity equation (3) is completed with the boundary conditions describing the firing and reset mechanisms of the network model (1), which reads as

$$
\lim _{V \rightarrow-\infty} \mathcal{F}^{(l)}(V \mid \eta, t)=\lim _{V \rightarrow \infty} \mathcal{F}^{(l)}(V \mid \eta, t) .
$$

Following the theoretical framework developed in ${ }^{38}$, one can apply the Ott-Antosen ansatz ${ }^{41}$ to obtain an exact macroscopic description of the infinite dimensional 2 -population system (1) in terms of collective variables. In order to obtain exact analytic results it is crucial to assume that the distribution of the neuronal excitabilities is a Lorentzian PDF, however the overall picture is not particularly influenced by considering others PDFs, like Gaussian and Erdös-Renyi ones ${ }^{38}$. In the case of the QIF model the macroscopic variables for each $l$-population are the instantaneous firing rate $r^{(l)}$, the average membrane potential $v^{(l)}$ and the mean synaptic activity $s^{(l)}$, which evolve according to:

$$
\begin{aligned}
& \dot{r}^{(l)}=\frac{\Delta^{(l)}}{\tau^{2} \pi}+\frac{2 r^{(l)} v^{(l)}}{\tau} \\
& \dot{v}^{(l)}=\frac{\left(v^{(l)}\right)^{2}+\bar{\eta}^{(l)}+I^{(l)}(t)}{\tau}+J_{l l} s^{(l)}+J_{k l} s^{(k)}-\tau\left(\pi r^{(l)}\right)^{2} \\
& \dot{s}^{(l)}=\frac{1}{\tau_{d}^{(l)}}\left[-s^{(l)}+r^{(l)}\right],
\end{aligned}
$$

for $l, k \in\{A, B\}$.

\section{Dynamical Indicators}

We make use of two dynamical indicators to characterize the evolution of the MF model (5): a Poincaré section and the corresponding Lyapunov Spectrum (LS).

The considered Poincaré section is defined as the manifold $\dot{r}^{(A)}(\bar{t})=0$ with $\dot{r}^{(A)}\left(\bar{t}^{-}\right)>0$, which amounts to identify the local maxima $r_{\max }$ of the time trace $r^{(A)}(t)$. On the other hand to compute the LS one should consider the time evolution of the tangent vector $\boldsymbol{\delta}=\left\{\delta r^{(A)}, \delta v^{(A)}, \delta s^{(A)}, \delta r^{(B)}, \delta v^{(B)}, \delta s^{(B)}\right\}$ resulting from the linearization of the original system Eq. (5), 
namely

$$
\begin{aligned}
\delta \dot{r}^{(l)} & =\frac{2\left(r^{(l)} \delta v^{(l)}+v^{(A)} \delta r^{(A)}\right)}{\tau} \\
\delta \dot{v}^{(l)} & =\frac{2 v^{(l)} \delta v^{(l)}}{\tau}+J_{l l} \delta s^{(l)}+J_{k l} \delta s^{(k)}-2 \pi^{2} \tau r^{(l)} \delta r^{(l)} \\
\delta \dot{s}^{(l)} & =\frac{\delta s^{(l)}+\delta r^{(l)}}{\tau_{d}^{(l)}}
\end{aligned}
$$

The LS is thus composed by 6 Lyapunov Exponents (LEs) $\left\{\lambda_{i}\right\}$ which quantify the average growth rates of infinitesimal perturbations along the different orthogonal manifolds estimated as

$$
\lambda_{i}=\lim _{t \rightarrow \infty} \frac{1}{t} \log \frac{|\boldsymbol{\delta}(t)|}{\left|\boldsymbol{\delta}_{0}\right|}
$$

by employing the well known technique described in Benettin et al. ${ }^{6}$ to maintain the tangent vectors orthonormal during the evolution. From the knowledge of the LS one can obtain an estimation of the fractal dimension of the corresponding invariant set in terms of the socalled Kaplan-Yorke dimension $D_{K Y}{ }^{29}$, defined implicitly as follows:

$$
\sum_{i=1}^{D_{K Y}} \lambda_{i} \equiv 0
$$

\section{Locking Characterization}

To investigate the capability of two interacting populations to lock their dynamics in a biologically relevant manner, we measure the degree of phase synchronization in the exactly reduced system (5). Therefore, we extract the phase of the population activity, by performing the Hilbert transform $\mathcal{H}[\cdot]$ of the firing rate for each population $l \in\{A, B\}$, thus obtaining the imaginary part of the analytic signal: namely, $\phi^{(l)}(t):=r^{(l)}(t)+j \mathcal{H}\left[r^{(l)}(t)\right]$. The evolution of the phase in time is then obtained as $\Phi^{(l)}(t)=\arg \left[\phi^{(l)}(t)\right]$. A generalized phase difference of the $n: m$ phase-locked mode can be defined as:

$$
\Delta \Phi_{n m}(t)=n \Phi^{(A)}-m \Phi^{(B)},
$$

and the degree of synchronization in the phase locked regime can be quantified in terms of the Kuramoto order parameter for the phase difference, namely:

$$
\rho_{n m}=\left|\left\langle e^{j \Delta \Phi_{n m}(t)}\right\rangle\right|
$$

where $\langle\cdot\rangle$ denotes a time average, and $|\cdot|$ is the norm of the complex number ${ }^{5,30}$.

\section{RESULTS}

\section{A. Self sustained oscillations in one population}

Firstly, by following ${ }^{20}$ we analyse the case of a single population with self-inhibition in absence of any external drive. Without lack of generality we take in account just population $A$ : this amounts to have a set of only three equations in (5) with $J_{B A}=I^{(A)}(t)=0$. For simplicity in the notation we finally drop the indices denoting the populations.

In the fully coupled QIF network oscillations can be observed only for IPSPs of finite duration, namely exponential in the present case. In particular, COs appear when the equilibrium point of the macroscopic system $\left(r_{0}, v_{0}, s_{0}\right)$ undergoes a Hopf bifurcation. Simulations of the QIF network model and the corresponding MF dynamics are compared in Fig. 1 revealing a very good agreement both in the asynchronous and in the oscillatory state. In particular, for the parameters considered in Fig. 1 the super-critical Hopf bifurcation takes place at $\tau_{d}^{(H)}=4.95 \mathrm{~ms}$ and panels Fig. 1 (A-C) refer to a stable focus for the MF at $\tau_{d}<\tau_{d}^{(H)}$, while panels Fig. 1 (D-E) to a stable limit cycle for $\tau_{d}>\tau_{d}^{(H)}$.

Due to the simplicity of the reduced model it is also possible to parametrize the Hopf boundaries where the asynchronous state loses stability as a function of the marginally stable solution $\left(r_{0}, v_{0}, s_{0}\right)$. In particular taking $\tau_{d}$ and $J$ as bifurcation parameters, one can verify that the boundaries of the Hopf bifurcation curves are defined by:

$$
\begin{aligned}
\tau_{d}^{(H)} & =\frac{9 \tau v_{0}^{2} \bar{\eta} \tau-\pi^{2} r_{0}^{2} \tau^{3} \pm \tau \sqrt{\left(\bar{\eta}-\pi^{2} r_{0}^{2} \tau^{2}\right)^{2}+2 v_{0}^{2}\left(9 \bar{\eta}-41 \pi^{2} r_{0}^{2} \tau^{2}\right)+17 v_{0}^{4}}}{16\left(\pi^{2} r_{0}^{2} \tau^{2} v_{0}+v_{0}^{3}\right)} \\
J^{(H)} & =\frac{\bar{\eta}+v_{0}^{2}}{r_{0} \tau}-\pi^{2} r_{0} \tau
\end{aligned}
$$

The equilibrium values are related by the equalities $v_{0}=-\Delta /\left(2 \tau \pi r_{0}\right)$ and $s_{0}=r_{0}$, with $r_{0}$ acting as a free parameter (see the Appendix for more details).

The phase diagrams showing the existence of self sus- 

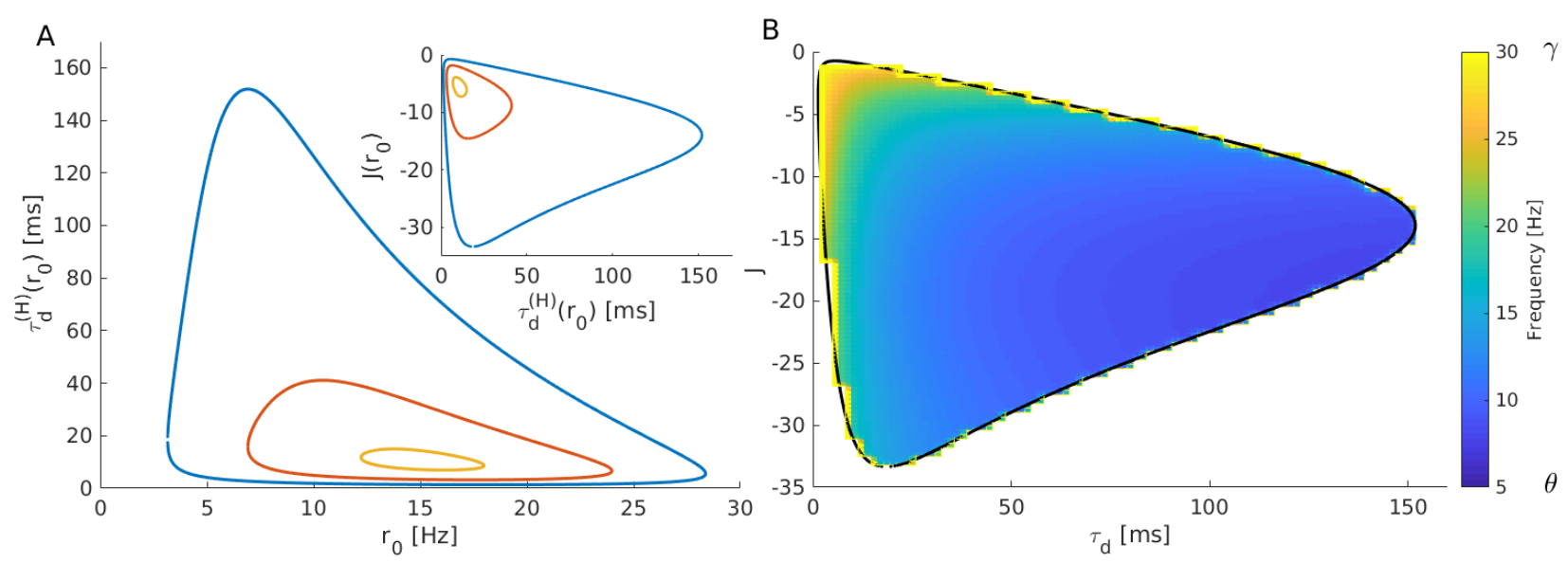

FIG. 2. Oscillation Stability Region: A) Hopf bifurcation boundaries in the $\left(r_{0}, \tau_{d}\right)$ plane obtained from (11) for three different values of the heterogeneity: namely $\Delta=0.05$ (blue), $\Delta=0.1$ (red), $\Delta=0.14$ (yellow). Inset: Hopf bifurcation boundaries in the $\left(\tau_{d}, J\right)$ space for the same $\Delta$-values. B) Heat map of the frequencies of oscillation of the instantaneous firing rate in the $\left(\tau_{d}, J\right)$ plane, for $\Delta=0.05$. The black curve coincides with the Hopf boundary denoted in blue in the inset of panel A). To quantify the frequencies of COs, a transient time $t_{t}=1 \mathrm{~s}$ is discarded and then the number of peaks in $r(t)$ are counted and divided by the simulation time $t_{s}=2 \mathrm{~s}$. For this figure, $\tau=10 \mathrm{~ms}$ and $\bar{\eta}=1$.

tained oscillations in the $\left\{r_{0}, \tau_{d}\right\}$ plane are displayed in Fig. 2 (A), for three values of $\Delta$ : the region inside the closed curves corresponds to the oscillating regime. Upon decreasing the dispersion of the excitability $(\Delta)$, the region of oscillatory behavior increases. This result highlights that some degree of homogeneity in the neural population is required in order to sustain a collective activity. In particular, for dispersions larger than a critical value $\Delta_{c}$ it is impossible for the system to sustain COs (for the parameter employed in the figure $\left.\Delta_{c} \approx 0.1453\right)$.

The inset in Fig. $2(\mathrm{~A})$ displays the same boundaries in the $\left\{\tau_{d}, J\right\}$ plane. From this figure one can observe that, upon increasing (decreasing) $\Delta$, the range of inhibitory strength and of synaptic times required to sustain oscillations decreases (increase). Thus indicating that more heterogeneous is the system the more the parameters $J$ and $\tau_{d}$ should be finely tuned in order to have COs. It's worth mentioning that for instantaneous synapses (corresponding to $\tau_{d} \rightarrow 0$ ) no oscillations can emerge autonomously in fully coupled systems with homogeneous synaptic coupling as shown in ${ }^{20}$ and evident from Fig. 2. However, COs can be observed in sparse balanced networks also for instantaneous synapses and in absence of any delay in the signal transmission ${ }^{21}$.

In order to understand the role played by the different parameters in modifying the frequency of the COs, we have estimated this frequency for $\Delta=0.5$ in the $\left(\tau_{d}, J\right)$ plane. The results are shown as a heat-map in Fig. 2 (B). It turns out that the frequency tends to decrease for increasing values of $\tau_{d}$ while it is almost independent on the value of $J$.

It is also worth noticing the fundamental role played by the self-inhibition in sustaining the autonomously generated oscillations, as it becomes clear from Eq. (11), since the Hopf bifurcations exist only for negative values of $J$.

From these results we can conclude that a single pop- ulation of QIF neurons can self sustain oscillations with a wide range of frequencies $\nu \simeq 5-30 \mathrm{~Hz}$ thanks to a finite synaptic time and to the self-inhibitory action of the neurons within the population.

\section{B. One population under external forcing}

Another relevant scenario in the framework of $\mathrm{CFC}^{25}$, is the case where an oscillatory drive $I(t)$ is applied to a neural population exhibiting COs. The forcing term can represent an input generated from an another neural population or an external stimulus. Therefore, we examine the behavior of the MF model driven by the following harmonic signal

$$
I(t)=-I_{0}\left(1+\sin \left(2 \pi \nu_{0} t\right)\right)
$$

characterized by a driving frequency $\nu_{0}$ and an amplitude $I_{0}$. Notice that we have chosen a strictly negative harmonic signal, to asses the effect on the population dynamics of a driving signal originating from a distinct inhibitory population.

The results of this analysis are illustrated in Fig. 3. First, we study the phase locking of the population dynamics, characterized by an oscillatory frequency $\nu$, to the modulatory input, for different forcing frequencies $\nu_{0}$ and amplitudes $I_{0}$ (see Fig. $3(\mathrm{~A})$ ). For small amplitudes, the external modulation is only able to lock the dynamics into a given $n: m$ mode (measured in terms of the indicator (10)) for a limited range of forcing frequencies $\nu_{0}$, while the ratio $n: m$ decreases for increasing $\nu_{0}$. Furthermore, the range where phase locking is observable increases with the amplitude $I_{0}$, thus giving rise to the Arnold tongues shown in Fig. 3 (A).

To better understand how the locking emerges, we consider the ratio $\nu / \nu_{0}$ between the $\mathrm{CO}$ frequency and the 

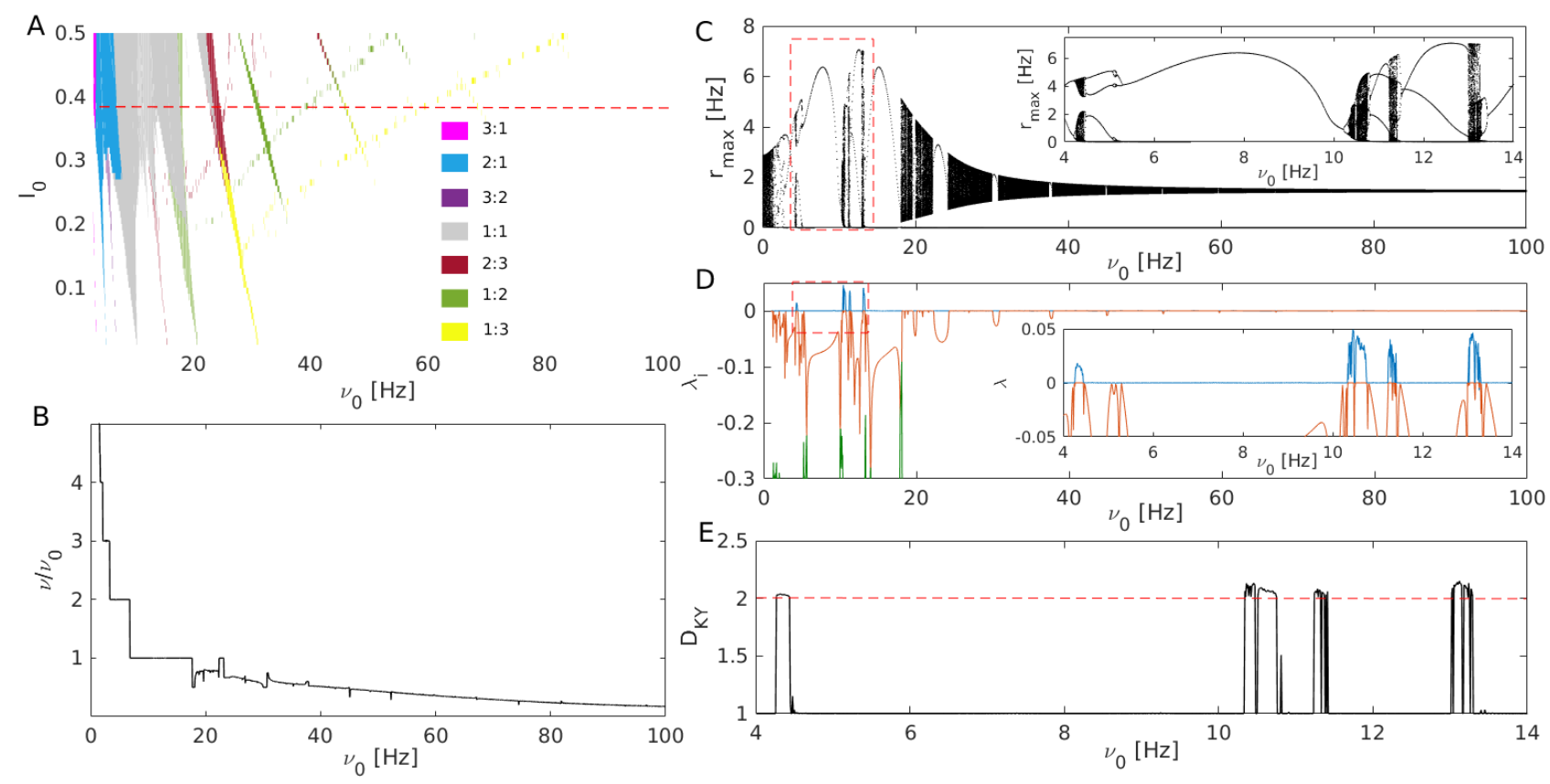

FIG. 3. Single population driven by a harmonic signal: A) Phase locking diagram of a single inhibitory population driven by a purely inhibitory harmonic external drive (13) in the $\left(I_{0}, \nu_{0}\right)$-plane. The Arnold's tongues corresponding to the different regions of phase locking are plotted in colors according to the color code reported in the panel. The dashed line indicates the value $I_{0}=0.4$ analysed in the subsequent panels. B) Ratio of the system's oscillation frequency $\nu$ and of the driving frequency $\nu_{0}$ as a function of $\nu_{0}$ showing a devil's staircase structure. Maxima of $r$ (C) and the corresponding Lyapunov exponents (D) as a function of $\nu_{0}$. In particular, the first, second and third LEs are reported as blue, red and green lines, respectively. The dashed rectangles indicate the zoomed regions in C) and D) shown in the corresponding insets. E) Kaplan-Yorke fractal dimension $D_{K Y}$ versus $\nu_{0}$, the dashed red line denotes a value of two. The employed parameters are $J=-10, \bar{\eta}, \Delta=0.01$, $\tau=10 \mathrm{~ms}, \tau_{d}=80 \mathrm{~ms}$. For the estimation of $r_{\max }$ and of the Lyapunov exponents a transient time of $t_{t}=1 \mathrm{~s}$ was discarded and then the maximum values were stored over a time interval $t_{s}=3 \mathrm{~s}$, while for the LS the tangent space was followed for a period $t_{s}=20 \mathrm{~s}$.

forcing one for a large interval of $\nu_{0}$-values and for a fixed amplitude value $I_{0}=0.4$, denoted as a red dashed line in Fig. 3 (A). The results are reported in Fig. 3 (B), the ratio $\nu / \nu_{0}$ reveals a structure similar to a devil's staircase, presenting plateaus (corresponding to the locked modes) intermingled with regions where the ratio has not always a monotonic behaviour. For the same parameters, we also report the maxima $r_{\max }$ of the instantaneous firing frequency of the forced system and the corresponding Lyapunov Spectrum (LS) in Figs. 3 (C) and (D), respectively. From these two indicators one can infer that most of the phase-locked regions correspond to regular periodic motion, as revealed by the single value of $r_{\max }$ and by a single zero LE observables in a large portion of the devil's staircase plateaus. On the other hand, for $\nu_{0}>15 \mathrm{~Hz}$ the regions where the $r(t)$ presents peaks of different heights are in correspondence with the nonflat regions of the devil's staircase. In particular these regions are associated to quasi-periodic motions, as confirmed by the existence of two zero LEs in the LS. A zoom in the region $\nu_{0}=[4,14] \mathrm{Hz}$, corresponding to $1: 1$ locking, is reported in the insets of Figs. $3(\mathrm{C})$ and (D). These enlargements show the emergence of chaotic windows, where $r_{\max }$ assumes values over continuous in- tervals and the maximal LE is positive. Furthermore, in these chaotic windows $D_{K Y}$ is slightly larger than two, as shown in $3(\mathrm{E})$, indicating that the chaotic attractor is low dimensional. This is confirmed by the stroboscopic attractor reported in Fig. 4 (B), obtained by reporting the macroscopic variables at regular time intervals equal to integer multiples of the forcing period $\nu_{0}^{-1}$.

Indeed the points of the attractor cover a set with a dimension slightly larger than one, since one degree of freedom is lost due to the stroboscopic observation. Interestingly, the chaotic motion appears despite the $1: 1$ locking, this means that the time trace of $r(t)$ presents always a single oscillation within a cycle of the external forcing but characterized by different amplitudes ${ }^{44}$, as shown in Fig. 4 (A).

\section{Two populations in a master-slave configuration}

Despite the fact that at a macroscopic level the network dynamics of a single population with exponential synapses is exactly described in the limit $N \rightarrow \infty$ by three degrees of freedom (5) our and previous analysis ${ }^{20}$ have not reported evidences of chaotic motions for a sin- 


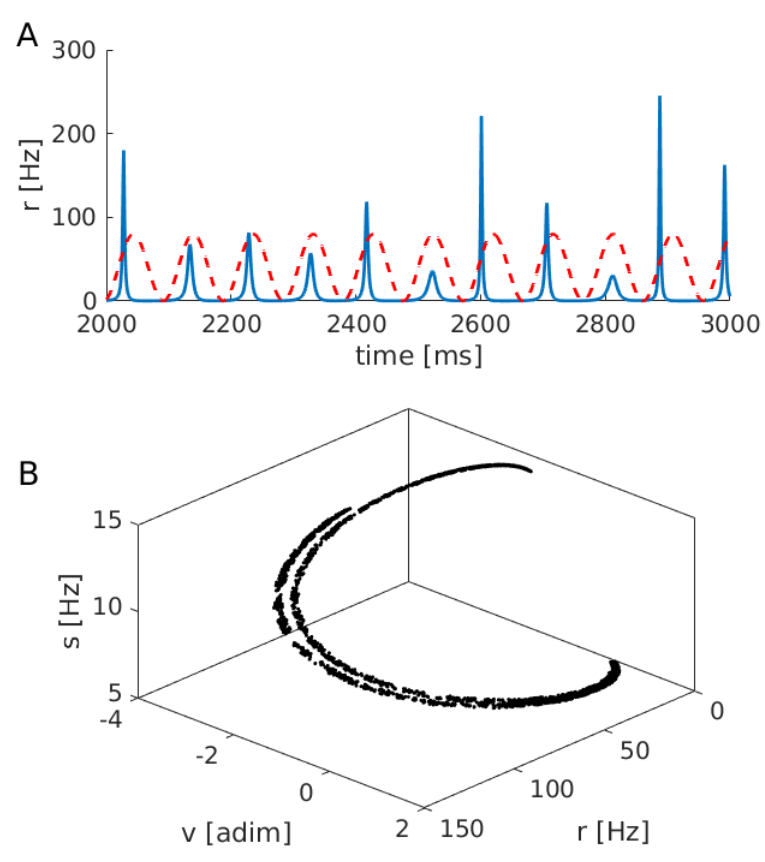

FIG. 4. Chaotic attractor for a single driven population: A) Time trace of the average firing rate $r(t)$ (blue solid line) and profile of the forcing term $I(t)$ (red dashed line) are shown for a chaotic regime.The chaotic dynamics is clearly locked to the frequency of the external drive. B) Stroboscopic attractor: the values of $(r, v, s)$ are recorded at regular time intervals corresponding to integer multiples of the forcing period $\nu_{0}^{-1}$. Parameters for this figure as in Fig. 3 with $\nu_{0}=10.4 \mathrm{~Hz}$. gle inhibitory population. The situation is different for an excitatory population, as briefly discussed in ${ }^{7}$, or in presence of an external forcing as shown in the previous sub-section.
In this sub-section we want to analyze the dynamical regimes emerging when a fast oscillating population (indicated as A) is driven by a a slowly oscillating population (denoted by B) in a master-slave configuration corresponding to $J_{B A} \neq 0$ and $J_{A B}=0$. Particular attention will be devoted to chaotic regimes. The different possible scenarios can be captured by considering only two sets of parameters, denoted as $\mathcal{C}_{1}$ and $\mathcal{C}_{2}$ and essentially characterized by different ratios of the synaptic time scales of the fast and slow family, namely:

$$
\begin{aligned}
& \mathcal{C}_{1}:=\left\{\tau_{d}^{(A)}=10 \mathrm{~ms}, J_{A A}=-10, \tau_{d}^{(B)}=50 \mathrm{~ms}, J_{B B}=-16\right\} \\
& \mathcal{C}_{2}:=\left\{\tau_{d}^{(A)}=2.5 \mathrm{~ms}, J_{A A}=-10, \tau_{d}^{(B)}=80 \mathrm{~ms}, J_{B B}=-20\right\}
\end{aligned}
$$

The coupling between the two population $J_{B A}$ and the network heterogeneity $\Delta=\Delta^{(A)}=\Delta^{(B)}$ will be employed as control parameters, while we will assume for simplicity $\bar{\eta}^{(A)}=\bar{\eta}^{(B)}=1$.

Let us first consider the set of parameters $\mathcal{C}_{1}$, in this case the analysis of the possible bifurcations arising in the $\left\{\Delta, J_{B A}\right\}$ plane reveals the existence of a codimension two bifurcation point at $\left(\Delta^{(H)}, J_{B A}^{(H)}\right) \approx(0.078,-4.75)$ which organizes the plane in four different regions. In these regions, labelled I-IV, the prevalent dynamics corresponds to stable foci in I, stable limit cycles in II and III, and to stable Tori $T^{2}$ in IV (see Fig. 5 (A) ). For each region we report in Fig. 5 (B) a corresponding sample trajectory projected in the sub-space $\left\{r^{(A)}, v^{(A)}\right\}$ taken in proximity of the codimension two point.

The critical vertical line observable at $\Delta^{(H)} \simeq 0.077$ in Fig. $5(\mathrm{~A})$ is a direct consequence of the super-critical Hopf bifurcation already present at the level of single population discussed in sub-section III A. This line for $J<J_{B A}^{(H)}$ is the locus of Hopf bifurcations (black solid) dividing foci (I) from stable oscillations (III), while at larger coupling $J_{B A}$ it becomes a secondary Hopf (or Torus) bifurcation line (blue solid) separating periodic (II) from quasi-periodic motions (IV). Moreover, the region III of stable limit cycles is divided by the region IV where Tori $T^{2}$ emerge from an another Torus bifurcation line (blue solid). Finally regions I and II are separated by a super-critical Hopf line (black solid).

We will now focus on the case $\Delta=0.01$ (corresponding to the red dashed line in Fig. $5(\mathrm{~A})$ ), to analyze the different regimes observable by varying $J_{B A}$. To this aim, similarly to what done in the previous sub-sections, we characterize the dynamics of the system in terms of the values of the Poincaré map $r_{\max }^{(A)}$ and of the associated LS. In particular in Fig. $6(\mathrm{~A})$ are reported the values of $r_{\max }^{(A)}$ in the range of cross-inhibition $J_{B A}=[-10,0]$. At very 
negative values of the cross-coupling we observe a single value for $r_{\text {max }}^{(A)}$, which corresponds to periodic COs. This is confirmed by the values of the LS reported in $6(\mathrm{~B})$ : the LEs are all negative except for the first one that is zero. At $J_{B A} \approx-6.05$ a broad band appears for the distribution of $r_{m a x}^{(A)}$ indicating that the time trace now displays maxima of different heights. This is due to the Torus bifurcation leading from a periodic to a quasi-periodic motion. The emergence of quasi-periodic motions is confirmed by the fact that in the corresponding intervals the first two LEs are zero (Fig. 6 (D)). For larger values of the cross-coupling (namely, $J_{B A} \approx[-5.8:-5]$ ) a period three window is clearly observable. Beyond this interval one observes quasi-periodic motions for almost all the negative values of the cross-coupling, apart narrow parameter intervals were locking of the two frequencies of the COs occur, as expected beyond a Torus bifurcation ${ }^{31}$.

We then proceed to study the parameter set $\mathcal{C}_{2}$. First, we show that the bidimensional bifurcation diagram in the plane $\left\{\Delta, J_{B A}\right\}$ presents a similar structure to that reported in Fig. $5(\mathrm{~A})$. Indeed also in the present case a codimension two point located at $\left\{\Delta^{(H)}, J_{B A}^{(H)}\right\} \approx$ $\{0.06,0\}$ divides the phase space in 4 regions analogous to those observed for the parameter set $\mathcal{C}_{1}$ and separated by the same kind of bifurcations (see the inset of Fig. $7(\mathrm{~A}))$. As in the previous case, we select the value $\Delta=0.01$ for analyzing the distribution of maxima of the firing rate $r^{(A)}$ and the associated Lyapunov spectra , see Fig. 7. For highly negative values of the crossinhibition we observe a periodic behaviour of the firing rate $r^{(A)}$. At the intersection with the torus bifurcation (occurring at $J_{B A}=-7.48$ ) quasi-periodicity emerges similarly to what observed for the parameter set $\mathcal{C}_{1}$. However, at larger values of the cross-inhibition (namely $J_{B A} \in[-7.3445,-7.3217]$ ) we observe a period-doubling cascade leading to chaos for $J_{B A} \simeq-7.32$. The systems stays chaotic in the interval $J_{B A} \in[-7.32,-7.18]$ apart for the occurrence of periodic windows. This is confirmed by the fact that the maximal LE becomes positive in the corresponding interval, as shown in Fig. 7 (D). An example of chaotic attractor is reported in Fig. 7 (F) with a fractal dimension slightly larger than two, as confirmed also by the estimation of $D_{K Y}$ whose values are displayed in Fig. $7(\mathrm{E})$. As in the case of the single forced population the macroscopic chaotic attractor is low dimensional.

For $J_{B A}>-7.18$ we have periodic and quasi-periodic activity, but no more chaos, in particular for $J_{B A}>-4.2$ we essentially observe mostly quasi-periodic motions up to $J_{B A}=0$. As mentioned before, the main difference between the parameter sets $\mathcal{C}_{1}$ and $\mathcal{C}_{2}$ is the ratio of the time scales associated to the synaptic filtering. For the parameter set $\mathcal{C}_{1}$ the time scale ratio $\tau_{d}^{(A)} / \tau_{d}^{(B)}$ is 1:5, while for the set $\mathcal{C}_{2}$ becomes 1:32. We have been able to find chaotic motions only for inhibitory populations with this large difference in their synaptic time scale. However, these values are biologically plausible, indeed they can correspond to populations of interneu-
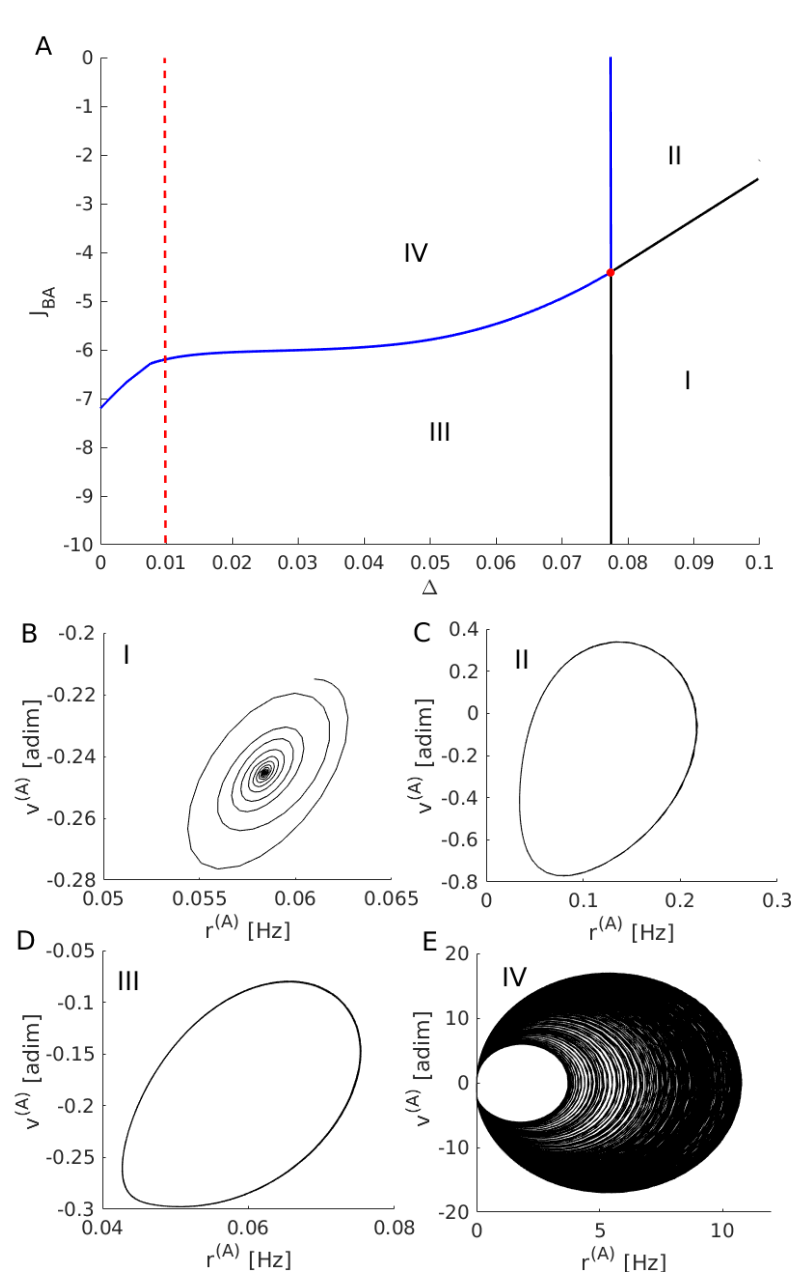

FIG. 5. Bifurcation diagram for the parameter set $\mathcal{C}_{1}$. A) Bifurcation diagram in the plane $\left(\Delta, J_{B A}\right)$ for the masterslave coupled system. The codimension 2 bifurcation point (red circle) divides the plane in 4 different regions (I-IV) corresponding to different dynamical regimes. The Hopf bifurcations (black lines) separate foci from limit cycles, while the Torus bifurcations (blue lines) denote the emergence of Tori $T^{2}$ from limit cycles. A sample trajectory for each one of the four regions are shown in panels (B-E). The red dashed line in (A) indicates the value of $\Delta$ considered in Fig. 6 .

rons generating IPSPs mediated via $\mathrm{GABA}_{A, \text { fast }}$ and $\mathrm{GABA}_{A, \text { slow }}$ receptors ${ }^{1}$, which have been identified in the hippocampus ${ }^{4}$ and in the cortex ${ }^{47}$.

\section{Cross-frequency-coupling in bidirectionally coupled populations}

As already mentioned a fundamental example of CFC, is represented by the coupling of the $\theta$ and $\gamma$ rhythms. Gamma oscillations are usually modulated by theta oscillations during locomotory actions and rapid eye movement (REM) sleep in the hippocampus ${ }^{34}$ as well as in the neocorte ${ }^{48}$. While gamma oscillations have been shown 

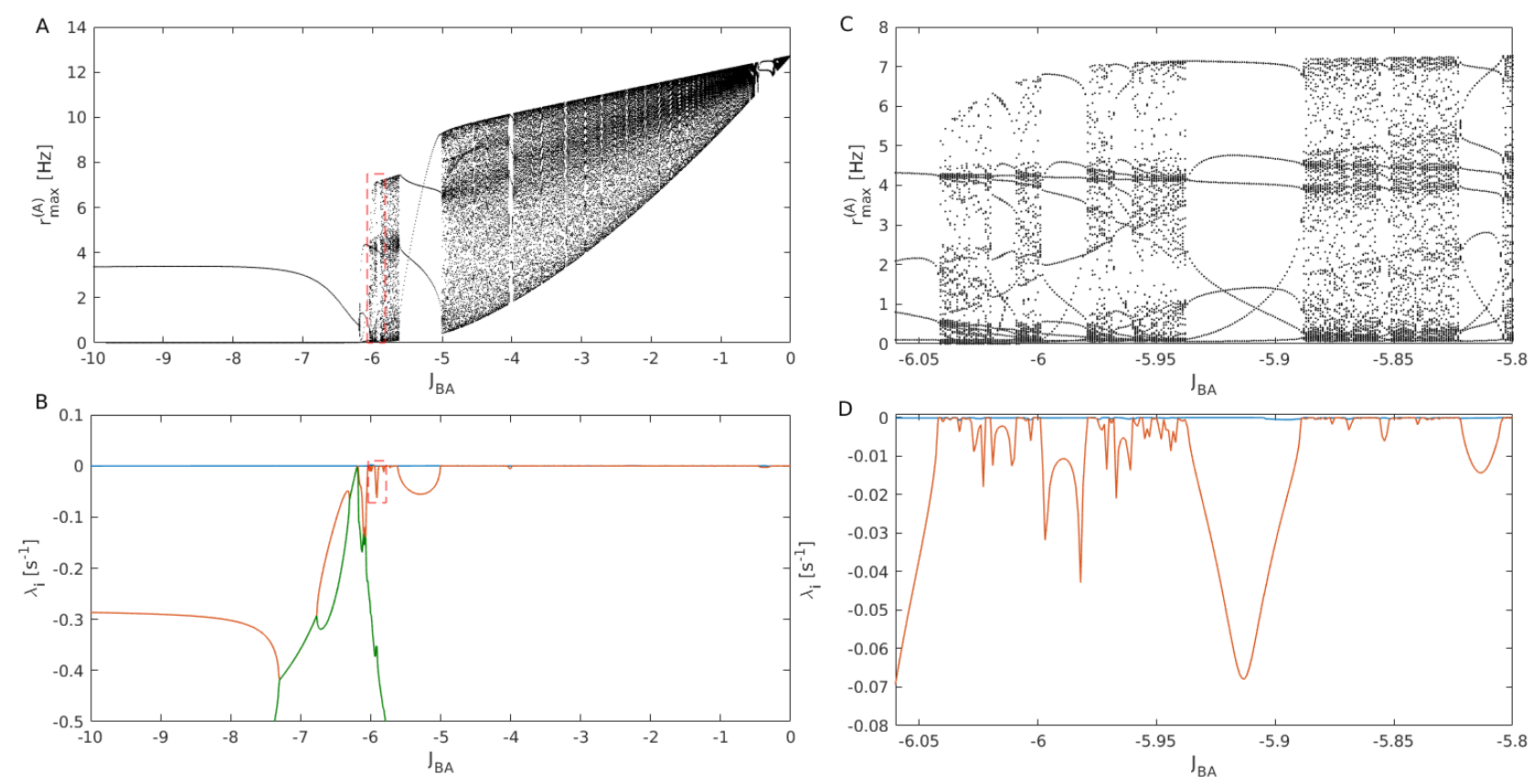

FIG. 6. Characterization of the dynamics for the parameter set $\mathcal{C}_{1}$. A) - C) Local maxima $r_{\max }^{(A)}$ of the firing rate of the A-population and B) - D) LEs as a function of the coupling strength $J_{A B}$. In panels (B,D) the blue curve represents the first LE, the orange the second LE and the green one the third LE. The dashed rectangle in panel A (B) indicates the zoomed region presented in panel C (D). For the evaluation of the maxima, a transient time of $t_{t}=10 \mathrm{~s}$ was discarded and then maximum values were stored during $t_{s}=15 \mathrm{~s}$. For the LEs estimation, after discarding the transient time $t_{t}$, the evolution of the tangent space was followed for a period $t=500 \mathrm{~s}$.

to be crucially dependent on inhibitory networks ${ }^{14}$, the origin of the $\theta$-modulation is still under debate. It has been suggested to be due either to an external excitatory drive $\mathrm{e}^{12}$ or to a cross-disinhibition originating from a distinct inhibitory population ${ }^{23,54}$.

In this sub-section we analyze the possibility that two bidirectionally interacting inhibitory populations could be at the basis of the $\theta-\gamma$ CFC. Inspired by previous analysis, we propose the difference in the synaptic time kinetics as a possible mechanism to achieve $\mathrm{CFC}^{54}$. Therefore we set the synaptic time scale of the fast (slow) population to $\tau_{A, d}=9 \mathrm{~ms}\left(\tau_{B, d}=50 \mathrm{~ms}\right)$, which corresponds approximately to the time scales of IPSPs generated via $\mathrm{GABA}_{A, \text { fast }}\left(\mathrm{GABA}_{A, \text { slow }}\right)$ receptors. Regarding the other parameters, internal to each population, these are chosen in a such a way that the self-generated oscillations correspond roughly to $\theta$ and $\gamma$ rhythms, respectively.

Firstly, we consider the case in which no external modulation is present, i.e $I^{(l)}(t)=0$. Depending on the value of the cross-coupling parameters $J_{A B}$ and $J_{B A}$ different type of $m: n$ P-P coupling can be achieved. In particular, as shown in Fig. 8 (A), we observe $1: 1$ and $2: 1$ phase synchronization in large regions of the $\left(J_{A B} ; J_{B A}\right)$ plane, while $3: 1$ and $5: 2$ locking emerge only along restricted stripes of the plane. In particular, we focus on the values of cross-inhibition $J_{l k}$ for which it is possible to achieve a $3: 1$ phase synchronization, corresponding to a $\theta-\gamma$ coupling. As evident from the green area in Fig. 8 (A), this specific P-P coupling occurs only for low values of $J_{A B}$, namely $J_{A B} \in[0.5,2.5]$.

Among the parameter values corresponding to $3: 1$ locking we choose for further analysis the ones for which the order parameter $\rho_{31}$ is maximal (denoted as a red circle in Fig. 8 (A)). In particular, we performed simulation of the network (1) as well as of the corresponding MF model (5). The raster plot in Fig. 8 (B) confirms that the two populations display COs locked in a $3: 1$ fashion. During the burst emitted from the slow population the fast one displays irregular asynchronous activity followed by three rapid bursts (each lasting around 10 $\mathrm{ms}$ ), before the next $\mathrm{CO}$ of the slow population. The fact that the slow population emits bursts of longer duration is confirmed by the analysis of the instantaneous firing rates reported in Fig. $8(\mathrm{D})$. Indeed, $r^{(A)}$ has oscillation of amplitude much larger than $r^{(B)}$ indicating that more neurons are recruited for a burst of population (A) with respect to population (B). This difference in the oscillations amplitude can also explain why the $3: 1$ locked mode is observable for $J_{A B} \ll J_{B A}$, indeed for larger $J_{A B}$ the activity of the slow population would be silenced. It is worth to notice in Fig. 8 (D) the good agreement between the firing rates obtained from the network simulations (dots) and from the evolution of the MF model (line).

An analysis of the power spectrum of $r^{(A)}$ (shown in 

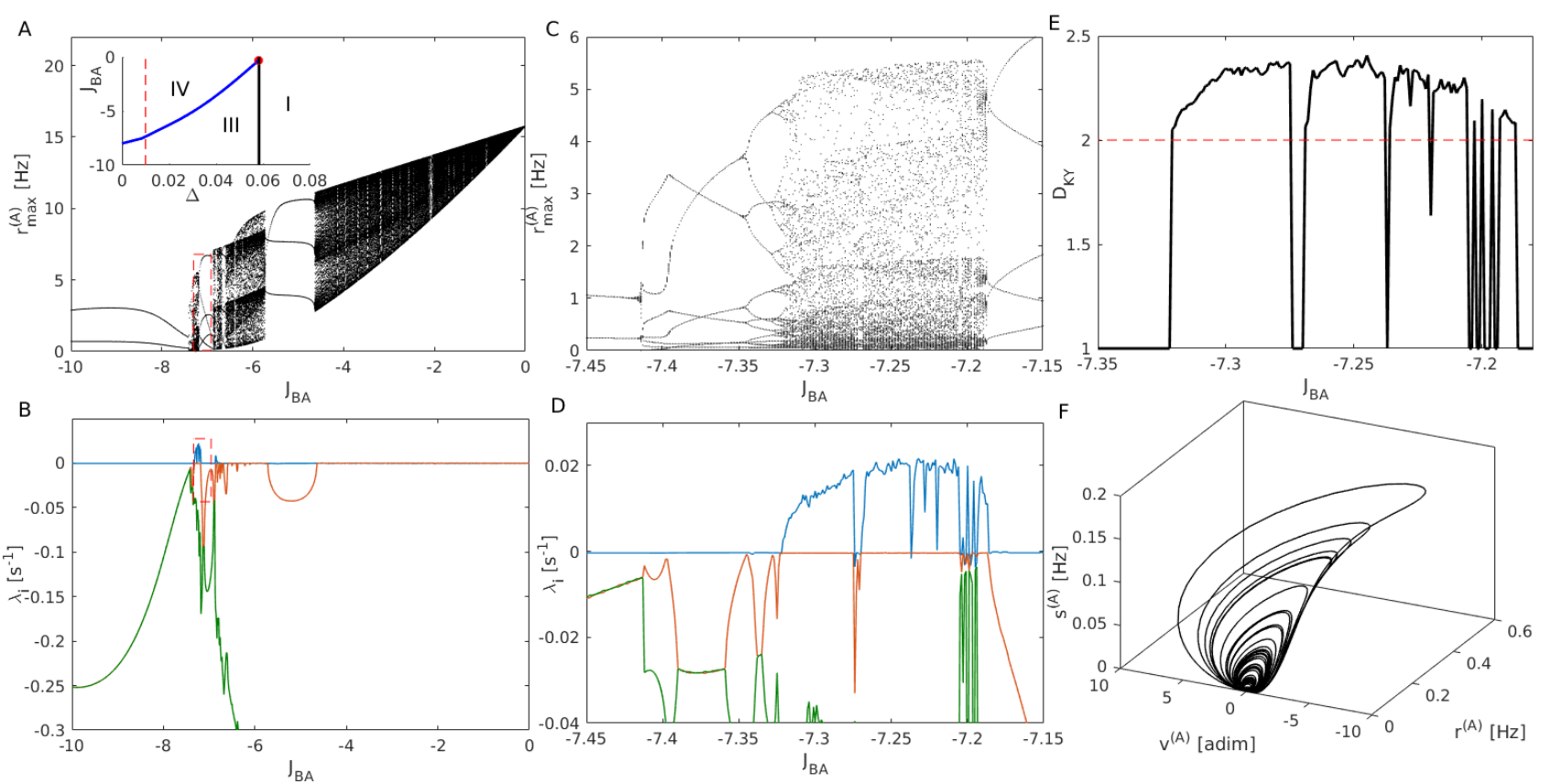

FIG. 7. Characterization of the dynamics for the parameter set $\mathcal{C}_{2}$. A-C) Values of $r_{\max }^{(A)}$ as a function of $J_{B A}$. In the inset it is shown the bifurcation diagram in the $\left(\Delta, J_{B A}\right)$ plane. This reveals the same structure of the diagram in Fig. 5 (A), here region (II) is not shown since it corresponds to the region of excitatory cross-coupling. B-D) First three LEs as a function of cross-inhibitory coupling $J_{B A}$. The red dashed rectangle in panel A (B) denotes the zoomed region presented in panel C (D). E) Kaplan-Yorke dimension $D_{K Y}$ in the parameter interval where chaos is present. F) Chaotic attractor in the $\left(r^{(A)}, v^{(A)}, s^{(A)}\right)$ space at $J_{B A}=-7.25$. For the evaluation of $r_{\text {max }}^{(A)}$, a transient time of $t_{t}=10 \mathrm{~s}$ was discarded and then maximal values were stored during $t_{s}=15 \mathrm{~s}$. For the LEs estimation, after the same transient time as before, the evolution of the tangent space was followed during $t=50 \mathrm{~s}$.

Fig. 8 (D)) reveals that the amount of power in the $\theta$ band is quite small (see the peak around $10 \mathrm{~Hz}$ in the inset) with respect to the power in the $\gamma$ band. This indicates that a CFC among the two bands is indeed present, but the interaction is limited.

Furthermore, varying the amplitude of the heterogeneity, as measured by $\Delta=\Delta^{(A)}=\Delta^{(B)}$, we can verify the capability of the network to sustain the $3: 1$ locked mode even in presence of disorder in the neural excitabilities. It can be seen that the system loses the ability to sustain such a locked state already for $\Delta>0.1$, indicating that CFC can occur only for a limited amount of disorder in the distribution of the neuronal excitabilities in agreement with the results reported $\mathrm{in}^{54}$ (see Fig. 8 (E). For large disorder the only possible locked state is that corresponding to $1: 1$ phase synchronization.

Sofar we have analyzed the possibility that $\theta$ and $\gamma$ rhythms were locally generated in inhibitory populations with different synaptic scales. However, the results of several optogenetic experiments performed for different area of the hippocampus and of the enthorinal cortex suggest that a $\theta$ frequency drive is sufficient to induce in vitro $\theta-\gamma \mathrm{CFCs}^{2,10,11,42}$. However, the interpretation of these experiments disagrees on the origin of the locally generated $\gamma$ oscillations. Two mechanism have been suggested: namely, either inhibitory ${ }^{2,42}$ or excitatory- inhibitory feedback loops ${ }^{10,11}$. Therefore, to clarify if recurrently coupled inhibitory populations, with different synaptic time scales, under a $\theta$-drive can display $\theta-\gamma$ $\mathrm{CFC}$ we drive the slow population via an external current $I^{(B)}=I_{0}^{(B)} \sin \left(2 \pi \nu_{\theta} t\right)$ with $\nu_{\theta}=10 \mathrm{~Hz}$, while the rest of the parameters remains unchanged.

As before, we look for the range of cross-inhibitions in which a $3: 1$ phase-locked mode emerges: results are plotted in Fig. 9 (A). We observe that the region where $\theta-\gamma \mathrm{CFC}$ can be observed definitely enlarge in presence of an external $\theta$-modulation. Furthermore, as observable from the power spectrum reported in Fig. 9 (B) the power in the $\theta$ band is noticeably increased as a consequence of the external modulation with respect to the non-modulated case. Moreover, the $\theta-\gamma$ CFC is now observable over a wider range of disorder on the excitabilities, indeed the $3: 1$ locked state survives up to $\Delta \approx 0.2-0.3$, as shown in Fig. $9(\mathrm{C})$. These evidences are similar to what reported $\mathrm{in}^{54}$, where adding a slow modulation to the $\theta$ generating population was sufficient to render more robust the observed CFC to the presence of disorder in the excitability distribution.

Finally, if we look at the network activity we observe two different scenarios corresponding to the $3: 1$ locked mode: (1) a P-P locking at low disorder and (2) a P-A locking (or $\theta$-nested $\gamma$ oscillations) at larger disorder. The 

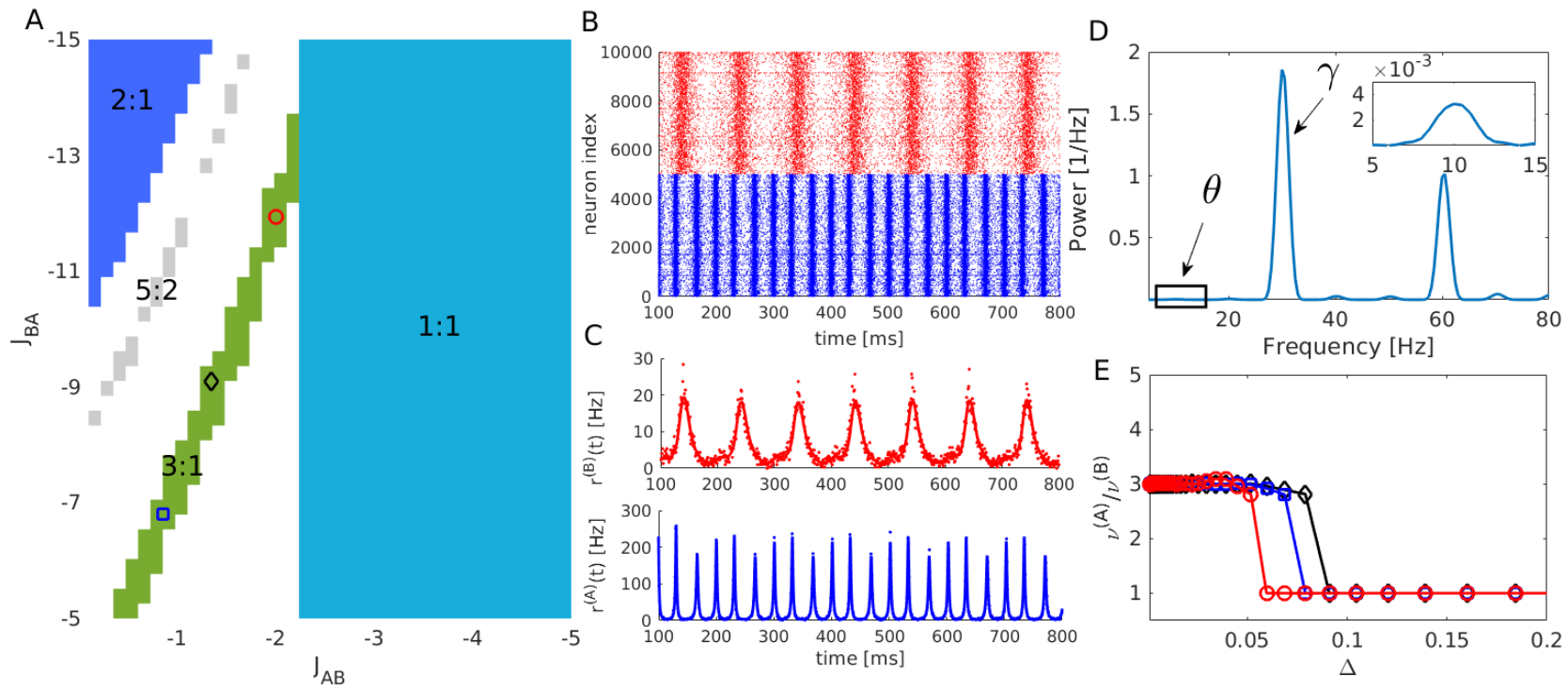

FIG. 8. CFC in bidirectionally coupled populations. A) Heat map of the order parameter locking modes for different values of the cross-coupling. Colored symbols denote three couple of parameters $\left\{J_{A B}, J_{B A}\right\}$ corresponding to $3: 1$ locking examined in (E) for different disorder values. In particular the red circle denotes the couple $\left\{J_{A B}, J_{B A}\right\}$ for which one obtains the maximal value of $\rho_{31}$ and these parameter values are employed for the simulations reported in (B,C). B) Raster plot of the network model Eq. (1) showing the fast and slow population in blue and red colors respectively. C) Instantaneous firing rates of the two populations obtained from the evolution of the MF dynamics (5) (same color code as in panel (B)). D) Power spectrum of the time trace $r^{(A)}(t)$ shown as a blue line in panel $(\mathrm{C})$, in the inset an enlargement of the spectrum corresponding to the $\theta$-band is shown. E) Ratio of the fast and slow frequencies of the COs $\nu^{(A)} / \nu^{(B)}$ showing the extent of the $3: 1$ locking interval for the three values of $\left\{J_{A B}, J_{B A}\right\}$ denoted by the symbols of the same color in (A) versus the disorder $\Delta:=\Delta_{A}=\Delta_{B}$. Parameters for fast population are $\tau_{d}^{(A)}=9 \mathrm{~ms}, \bar{\eta}_{A}=2, J_{A A}=-2$ and for the slow one $\tau_{d}^{(B)}=50 \mathrm{~ms}, \bar{\eta}_{B}=1.5, J_{B B}=-18$. Common parameters $\Delta=0.05$ (for panels A to D) and $\tau=10$. The time traces used for the phase locking analysis were taken over a period of $t=10 \mathrm{~s}$ after discarding an initial transient time $t_{t}=10 \mathrm{~s}$. For the network simulations in B), $N_{A}=N_{B}=10000$ neurons, in the figure only half of them are depicted. The spectrum in (D) was obtained by averaging 50 power spectra each calculated over a time trace of duration $t=16.384 \mathrm{~s}$ with $2^{15}$ equispaced samples, after a transient time of $t_{t}=10 \mathrm{~s}$.

first scenario is characterized by the fast population displaying clear COs slightly modulated in their amplitudes by the activity of the slow population, but tightly locked in phase with the slow ones (see Fig. 10 (A,B)). The second scenario presents a firing activity of the fast population strongly modulated in its amplitude by the slow population as observable in Fig. $10(\mathrm{~A}, \mathrm{~B})$. In this latter case the neurons in population (A) fire almost asynchronously with a really low firing rate, however the coupling with by the activity of the slow population (B) is reflected in a clear modulation of the firing rate $r^{(A)}$, analogously to what reported for $\theta$-nested $\gamma$ oscillations induced by optogenetic stimulation ${ }^{11,42}$.

\section{CONCLUDING REMARKS}

Self sustained oscillatory behavior has been widely studied in the neuroscientific community, as several coding mechanisms rely on the rhythms generated by interneuronal populations ${ }^{53}$. In particular, the emergence of COs in inhibitory networks has been usually related to the presence of an additional timescale, beyond the one associated with the membrane potential evolution, which can be either the transmission delay ${ }^{8,9}$ or a finite synaptic time ${ }^{18,20,36,51}$. The only example of COs emerging for instantaneous synapses and in absence of delay has been reported in ${ }^{21}$ for a sparse network of QIF neurons in a balanced setup. In this case, for values of the in-degree sufficiently large the COs emerge due to endogenous fluctuations, which persist in the thermodynamic limit.

In this paper we have considered an heterogeneous inhibitory population with exponentially decaying synapses, which can be described at a macroscopic level by an exact reduced model of three variables: namely, the firing rate, the average membrane potential and the mean synaptic activity. As shown in $^{18,20}$, the presence of the synaptic dynamics is at the origin of the COs, emerging via a super-critical Hopf bifurcation. In particular, we have shown that the period of the COs is controlled by the synaptic time scale and that, for increasing heterogeneity, the observation of COs require finer and finer tuning of the model parameters. Moreover, we have characterized in detail the effect of an in- 
hibitory periodic current on a single self-oscillating population. The external forcing leads to the appearance of locking phenomena characterized by Arnold tongues and devil's staircase. We have also identified low dimensional chaotic windows, where the instantaneous firing rate of the forced population display oscillations of irregular amplitudes, but tightly locked to the external signal oscillations ${ }^{44}$.

Furthermore, we considered two inhibitory populations connected in a master-slave configuration, i.e. unidirectionally coupled, where the fast oscillating population is forced by the one with slow synaptic dynamics. In a single population we observed, for sufficiently large heterogeneity, only focus solutions at the macroscopic level, which can turn in COs by reducing the disorder in the network. In presence of a second population the complexity of the macroscopic solutions increases for crossinhibitory coupling not too negative. In particular, by increasing the cross-coupling, the focus becomes a limit cycle via a super-critical Hopf bifurcation and the COs a quasi-periodic motion via a Torus bifurcation. Depending on the parameter values a period doubling cascade leading to chaotic behaviour is observable above the Torus bifurcation. In particular, the macroscopic attractor has a fractal dimension slightly larger than two with a single associated positive Lyapunov exponent despite the fact that the two coupled neural mass model are described by six degrees of freedom. The macroscopic solutions we have found in the master-slave configuration are similar to those identified in $^{37}$ for $\theta$-neurons populations coupled via pulses of finite width.

Even though the dichotomy between chaos and reliability in brain coding is a debated topic ${ }^{3,26,32,35}$, it is of great importance to establish the conditions in which such behavior may appear. In the present case, chaotic behavior is found only when the synaptic time scales of the two inhibitory populations are quite different. However, these values are consistent with synaptic times observable in interneuron populations with fast and slow $\mathrm{GABA}_{A}$ receptors, as shown in ${ }^{4,47,54}$.

We finally explored the possibility that two interacting inhibitory networks could give rise to specific crossfrequency-coupling mechanisms ${ }^{25}$. In particular, for its relevance in neuroscience we limited the analysis to $\theta-\gamma$ CFC emerging as a consequence of the interaction between fast and slow $\mathrm{GABA}_{A}$ kinetics, as reported in ${ }^{54}$ for populations of Hodgkin-Huxley neurons. In the original set-up was possible to observe $\theta-\gamma$ CFC coupling in a narrow region of parameters and for a limited range of heterogeneity. The addition of a $\theta$-forcing on the slow population renders the system more robust to the disorder in the excitability distribution and enlarges the observability region of the $\theta-\gamma$ CFC. Furthermore, we observed two kind of CFC: namely, P-P (P-A) coupling for low (high) heterogeneity. Both these scenarios have been reported experimentally for $\theta-\gamma$ oscillations: namely, P-A coupling has been reported in vitro for optogenetic $\theta$-stimulations of the the hippocampal area $\mathrm{CA} 1^{11}$ and $\mathrm{CA} 3^{2}$, as well as of the medial enthorinal cortex ${ }^{42}$; P-P coupling have been observed in the hippocampus in behaving rats ${ }^{5,17}$.

Our analysis shows, for the first time to our knowledge, that the $\theta-\gamma$ CFC, reported for Hodgkin-Huxley networks in ${ }^{54}$, can be reproduced also at the level of exact neural mass models for two coupled inhibitory populations. Our results pave the way for further studies of other CFC mechanisms present in the brain, e.g by employing analytically estimated macroscopic Phase Response Curves ${ }^{22}$ to characterize phase synchronization in multiscale networks of QIF neurons.

\section{ACKNOWLEDGMENTS}

Authors are in debt with Ernest Montbrió for various enlightening interactions in the first phase of development of this project, furthermore they acknowledge fruitful discussions with Federico Devalle, Boris Gutkin, and Alex Roxin. A.T. received financial support by the Excellence Initiative A*MIDEX (Grant No. ANR-11-IDEX-0001-02) (together with D. A.-G.), by the Excellence Initiative I-Site Paris Seine (Grant No ANR-16-IDEX-008), by the Labex MME-DII (Grant No ANR-11-LBX-0023-01) (together with S.O.) and by the ANR Project ERMUNDY (Grant No ANR-18-CE370014), all part of the French programme "Investissements d'Avenir". D.A-G was also supported by CNRS for a research period at LPTM, UMR 8089, Université de Cergy-Pontoise, France. A.C was supported by Erasmus+ Traineeship 2016/2017 contract between University of Florence, Department of Mathematics and Computer Science "Ulisse Dini" (DIMAI), and Centre de Physique Théorique (CPT) and LabEx Archimède, Marseille, France.

\section{APPENDIX: HOPF BOUNDARIES}

In the case of a single population of inhibitory neurons with no external input, the fixed point solutions $\left(v_{0}, r_{0}, s_{0}\right)$ of the MF model (5) are given by the following set of equations

$$
\begin{aligned}
v_{0} & =-\frac{\Delta}{2 \tau \pi r_{0}} \\
v_{0}^{2}+\bar{\eta}-\left(\pi \tau r_{0}\right)^{2}+\tau J s_{0} & =0 \\
s_{0} & =r_{0} .
\end{aligned}
$$

In order to study the linear stability of the equilibrium point, we consider the corresponding eigenvalue problem, namely

$$
\operatorname{det}\left[\begin{array}{ccc}
2 v_{0} / \tau-\Lambda & 2 r_{0} / \tau & 0 \\
-2 \pi^{2} \tau r_{0} & 2 v_{0} / \tau-\Lambda & J \\
1 / \tau_{d} & 0 & -\Lambda-1 / \tau_{d}
\end{array}\right]=0
$$

where $\Lambda$ are the complex eigenvalues which can be found 
by solving the following characteristic polynomial

$$
p(\Lambda)=\tau_{d} \tau^{2} \Lambda^{3}+A \Lambda^{2}+\Lambda\left(\tau_{d} B-4 \tau v_{0}\right)+\left(B-2 r_{0} J \tau\right)
$$

where $A=\left(\tau^{2}-4 v_{0} \tau_{d} \tau\right)$ and $B=\left(4 v_{0}^{2}+4 \pi^{2} r_{0}^{2} \tau^{2}\right)$.

In order to obtain a parametrization of the Hopf bifurcation curve we impose $\Lambda=i \Omega$ with $\Omega \in \Re \backslash\{0\}$ and solve $p(i \Omega)=0$, which can only be satisfied if

$$
\operatorname{Re}[p(i \Omega)]=0 \quad \text { and } \quad \operatorname{Im}[p(i \Omega)]=0 .
$$

Solving for $\Omega$ Eqs. (19) we end up with:

$$
\begin{aligned}
& \Omega_{\mathrm{Re}}= \pm \sqrt{\frac{\left(B-2 r_{0} J \tau\right)}{A}} \\
& \Omega_{\mathrm{Im}}= \pm \sqrt{\frac{\left(\tau_{d} B-4 \tau v_{0}\right)}{\tau_{d} \tau^{2}}} .
\end{aligned}
$$

By equating (20) and (21) we can find the values of $J^{(H)}$ where the Hopf bifurcation occurs, namely

$$
J^{(H)}=\frac{2 v_{0}\left[\tau^{2}\left(4 \pi^{2} \tau_{d}^{2} r_{0}^{2}+1\right)+4 \tau_{d}^{2} v_{0}^{2}-4 \tau_{d} \tau v_{0}\right]}{\tau_{d} r_{0} \tau^{2}} .
$$

Finally, by introducing Eq. (22) in (15), and noticing from Eq. (16) that $s_{0}=r_{0}$, one can derive the values of the synaptic time scale $\tau_{d}^{(H)}$ that bounds the oscillating region and that is reported in Eq. (11). Notice that the dependence on $\Delta$ is implicitly introduced in the expression of $v_{0}$, therefore, it possible to obtain also the critical value $\Delta^{(H)}$ associated to the Hopf transition by substituting (14) in (15) and solving for $\Delta$. It should be also stressed that this approach cannot distinguish between super-critical and sub-critical Hopf bifurcations. As a matter of fact for an inhibitory QIF population with exponential synapses no sub-critical bifurcations have been reported for homogeneous synaptic couplings ${ }^{20}$, while these emerge whenever a disorder is introduced either in the synaptic couplings or in the link distribution ${ }^{7}$.

${ }^{1}$ GABA (gamma-Aminobutyric acid) is the main inhibitory neurotransmitter in the adult mammalian brain, GABA performs its action by binding to $\mathrm{GABA}_{A}$ or $\mathrm{GABA}_{B}$ receptors.

${ }^{2}$ Akam, T., Oren, I., Mantoan, L., Ferenczi, E., and Kullmann, D. M. (2012). Oscillatory dynamics in the hippocampus support dentate gyrus-ca3 coupling. Nature neuroscience, 15(5):763.

${ }^{3}$ Angulo-Garcia, D. and Torcini, A. (2014). Stable chaos in fluctuation driven neural circuits. Chaos, Solitons \& Fractals, $69(0): 233-245$.

${ }^{4}$ Banks, M. I., Li, T.-B., and Pearce, R. A. (1998). The synaptic basis of gabaa, slow. Journal of Neuroscience, 18(4):1305-1317.

${ }^{5}$ Belluscio, M. A., Mizuseki, K., Schmidt, R., Kempter, R., and Buzsáki, G. (2012). Cross-frequency phase-phase coupling between theta and gamma oscillations in the hippocampus. Journal of Neuroscience, 32(2):423-435.

${ }^{6}$ Benettin, G., Galgani, L., Giorgilli, A., and Strelcyn, J.-M. (1980). Lyapunov characteristic exponents for smooth dynamical systems and for hamiltonian systems; a method for computing all of them. part 1: Theory. Meccanica, 15(1):9-20.

${ }^{7}$ Bi, H., Segneri, M., di Volo, M., and Torcini, A. (2019). Coexistence of fast and slow gamma oscillations in one population of inhibitory spiking neurons. arXiv preprint arXiv:190\%.00230.
${ }^{8}$ Brunel, N. (2000). Dynamics of sparsely connected networks of excitatory and inhibitory spiking neurons. J. Comput. Neurosci., 8(3):183-208.

${ }^{9}$ Brunel, N. and Hakim, V. (1999). Fast global oscillations in networks of integrate-and-fire neurons with low firing rates. Neural. Comput., 11(1):1621-1671.

${ }^{10}$ Butler, J. L., Hay, Y. A., and Paulsen, O. (2018). Comparison of three gamma oscillations in the mouse entorhinal-hippocampal system. European Journal of Neuroscience, 48(8):2795-2806.

${ }^{11}$ Butler, J. L., Mendonça, P. R., Robinson, H. P., and Paulsen, O. (2016). Intrinsic cornu ammonis area 1 theta-nested gamma oscillations induced by optogenetic theta frequency stimulation. Journal of Neuroscience, 36(15):4155-4169.

${ }^{12}$ Buzsáki, G. (2002). Theta oscillations in the hippocampus. Neuron, 33(3):325-340.

${ }^{13}$ Buzsaki, G. (2006). Rhythms of the Brain. Oxford University Press, USA, 1 edition.

${ }^{14}$ Buzsáki, G. and Wang, X.-J. (2012). Mechanisms of gamma oscillations. Annual review of neuroscience, 35:203.

${ }^{15}$ Canolty, R. T. and Knight, R. T. (2010). The functional role of cross-frequency coupling. Trends in cognitive sciences, 14(11):506-515.

${ }^{16}$ Capocelli, R. and Ricciardi, L. (1971). Diffusion approximation and first passage time problem for a model neuron. Kybernetik, 8(6):214-223.

${ }^{17}$ Colgin, L. L., Denninger, T., Fyhn, M., Hafting, T., Bonnevie, T., Jensen, O., Moser, M.-B., and Moser, E. I. (2009). Frequency of gamma oscillations routes flow of information in the hippocampus. Nature, 462(7271):353.

${ }^{18}$ Coombes, S. and Byrne, Á. (2019). Next generation neural mass models. In Corinto, F. and Torcini, A., editors, Nonlinear Dynamics in Computational Neuroscience, PoliTO Springer Series, pages 1-16. Springer, Cham.

${ }^{19}$ David, O. and Friston, K. J. (2003). A neural mass model for meg/eeg:: coupling and neuronal dynamics. NeuroImage, 20(3):1743-1755.

${ }^{20}$ Devalle, F., Roxin, A., and Montbrió, E. (2017). Firing rate equations require a spike synchrony mechanism to correctly describe fast oscillations in inhibitory networks. PLoS computational biology, 13(12):e1005881.

${ }^{21}$ di Volo, M. and Torcini, A. (2018). Transition from asynchronous to oscillatory dynamics in balanced spiking networks with instantaneous synapses. Physical review letters, 121(12):128301.

${ }^{22}$ Dumont, G., Ermentrout, G. B., and Gutkin, B. (2017). Macroscopic phase-resetting curves for spiking neural networks. Physical Review E, 96(4):042311.

${ }^{23}$ Hangya, B., Borhegyi, Z., Szilágyi, N., Freund, T. F., and Varga, V. (2009). Gabaergic neurons of the medial septum lead the hippocampal network during theta activity. Journal of Neuroscience, 29(25):8094-8102.

${ }^{24}$ Holz, E. M., Glennon, M., Prendergast, K., and Sauseng, P. (2010). Theta-gamma phase synchronization during memory matching in visual working memory. Neuroimage, 52(1):326-335.

${ }^{25}$ Hyafil, A., Giraud, A.-L., Fontolan, L., and Gutkin, B. (2015). Neural cross-frequency coupling: connecting architectures, mechanisms, and functions. Trends in neurosciences, 38(11):725-740.

${ }^{26}$ Jahnke, S., Memmesheimer, R.-M., and Timme, M. (2008). Stable irregular dynamics in complex neural networks. Phys. Rev. Lett., 100:048102.

${ }^{27}$ Jansen, B. H. and Rit, V. G. (1995). Electroencephalogram and visual evoked potential generation in a mathematical model of coupled cortical columns. Biological cybernetics, 73(4):357-366.

${ }^{28}$ Jensen, O. and Colgin, L. L. (2007). Cross-frequency coupling between neuronal oscillations. Trends in cognitive sciences, 11(7):267-269.

${ }^{29}$ Kaplan, J. and Yorke, J. (1979). Functional differential equations and approximation of fixed points. Lecture notes in mathematics, 730:204-227.

${ }^{30}$ Kuramoto, Y. (2012). Chemical oscillations, waves, and turbulence, volume 19. Springer Science \& Business Media. 
${ }^{31}$ Kuznetsov, Y. A. (2013). Elements of applied bifurcation theory, volume 112. Springer Science \& Business Media.

${ }^{32}$ Lajoie, G., Lin, K. K., and Shea-Brown, E. (2013). Chaos and reliability in balanced spiking networks with temporal drive. Phys. Rev. E, 87:052901.

${ }^{33}$ Lega, B., Burke, J., Jacobs, J., and Kahana, M. J. (2014). Slowtheta-to-gamma phase-amplitude coupling in human hippocampus supports the formation of new episodic memories. Cerebral Cortex, 26(1):268-278.

${ }^{34}$ Lisman, J. E. and Jensen, O. (2013). The theta-gamma neural code. Neuron, 77(6):1002-1016.

${ }^{35}$ London, M., Roth, A., Beeren, L., Häusser, M., and Latham, P. E. (2010). Sensitivity to perturbations in vivo implies high noise and suggests rate coding in cortex. Nature., 466:123-127.

${ }^{36}$ Luke, T. B., Barreto, E., and So, P. (2013). Complete classification of the macroscopic behavior of a heterogeneous network of theta neurons. Neural computation, 25(12):3207-3234.

${ }^{37}$ Luke, T. B., Barreto, E., and So, P. (2014). Macroscopic complexity from an autonomous network of networks of theta neurons. Frontiers in computational neuroscience, 8:145.

${ }^{38}$ Montbrió, E., Pazó, D., and Roxin, A. (2015). Macroscopic description for networks of spiking neurons. Physical Review X, $5(2): 021028$.

${ }^{39}$ Moreno-Bote, R. and Parga, N. (2010). Response of integrateand-fire neurons to noisy inputs filtered by synapses with arbitrary timescales: Firing rate and correlations. Neural Computation, 22(6):1528-1572.

${ }^{40}$ Olmi, S., Angulo-Garcia, D., Imparato, A., and Torcini, A. (2017). Exact firing time statistics of neurons driven by discrete inhibitory noise. Scientific Reports, 7(1):1577.

${ }^{41} \mathrm{Ott}$, E. and Antonsen, T. M. (2008). Low dimensional behavior of large systems of globally coupled oscillators. Chaos: An Interdisciplinary Journal of Nonlinear Science, 18(3):037113.

${ }^{42}$ Pastoll, H., Solanka, L., van Rossum, M. C., and Nolan, M. F. (2013). Feedback inhibition enables theta-nested gamma oscillations and grid firing fields. Neuron, 77(1):141-154.

${ }^{43}$ Pazó, D. and Montbrió, E. (2014). Low-dimensional dynamics of populations of pulse-coupled oscillators. Physical Review X, 4(1):011009

${ }^{44}$ Pikovsky, A., Zaks, M., Rosenblum, M., Osipov, G., and Kurths,
J. (1997). Phase synchronization of chaotic oscillations in terms of periodic orbits. Chaos: An Interdisciplinary Journal of Nonlinear Science, 7(4):680-687.

${ }^{45}$ Richardson, M. J. and Swarbrick, R. (2010). Firing-rate response of a neuron receiving excitatory and inhibitory synaptic shot noise. Physical review letters, 105(17):178102.

${ }^{46}$ ROSEnBlum, M., TASS, P., Kurths, J., VOLKMANN, J., SCHNITZLER, A., and FREUND, H.-J. (2000). Detection of phase locking from noisy data: application to magnetoencephalography. In Chaos In Brain?, pages 34-51. World Scientific.

${ }^{47}$ Sceniak, M. P. and MacIver, M. B. (2008). Slow gaba a mediated synaptic transmission in rat visual cortex. BMC neuroscience, $9(1): 8$.

${ }^{48}$ Sirota, A., Montgomery, S., Fujisawa, S., Isomura, Y., Zugaro, M., and Buzsáki, G. (2008). Entrainment of neocortical neurons and gamma oscillations by the hippocampal theta rhythm. Neuron, 60(4):683-697.

${ }^{49}$ Treves, A. (1993). Mean-field analysis of neuronal spike dynamics. Network: Computation in Neural Systems, 4(3):259-284.

${ }^{50}$ Ullner, E., Politi, A., and Torcini, A. (2019). Self-consistent analysis of asynchronous neural activity. in preparation.

${ }^{51}$ Van Vreeswijk, C., Abbott, L., and Ermentrout, G. B. (1994). When inhibition not excitation synchronizes neural firing. Journal of computational neuroscience, 1(4):313-321.

${ }^{52}$ Varela, F., Lachaux, J.-P., Rodriguez, E., and Martinerie, J. (2001). The brainweb: phase synchronization and large-scale integration. Nature reviews neuroscience, 2(4):229.

${ }^{53}$ Wang, X.-J. (2010). Neurophysiological and computational principles of cortical rhythms in cognition. Physiological reviews, 90(3):1195-1268.

${ }^{54}$ White, J. A., Banks, M. I., Pearce, R. A., and Kopell, N. J. (2000). Networks of interneurons with fast and slow $\gamma$-aminobutyric acid type a (gabaa) kinetics provide substrate for mixed gamma-theta rhythm. Proceedings of the National Academy of Sciences, 97(14):8128-8133.

${ }^{55}$ Wilson, H. R. and Cowan, J. D. (1972). Excitatory and inhibitory interactions in localized populations of model neurons. Biophysical journal, 12(1):1-24. 
bioRxiv preprint doi: https://doi.org/10.1101/745828; this version posted August 24,2019 . The copyright holder for this preprint (which was not certified by peer review) is the author/funder, who has granted bioRxiv a license to display the preprint in perpetuity. It is made available under aCC-BY-NC-ND 4.0 International license.
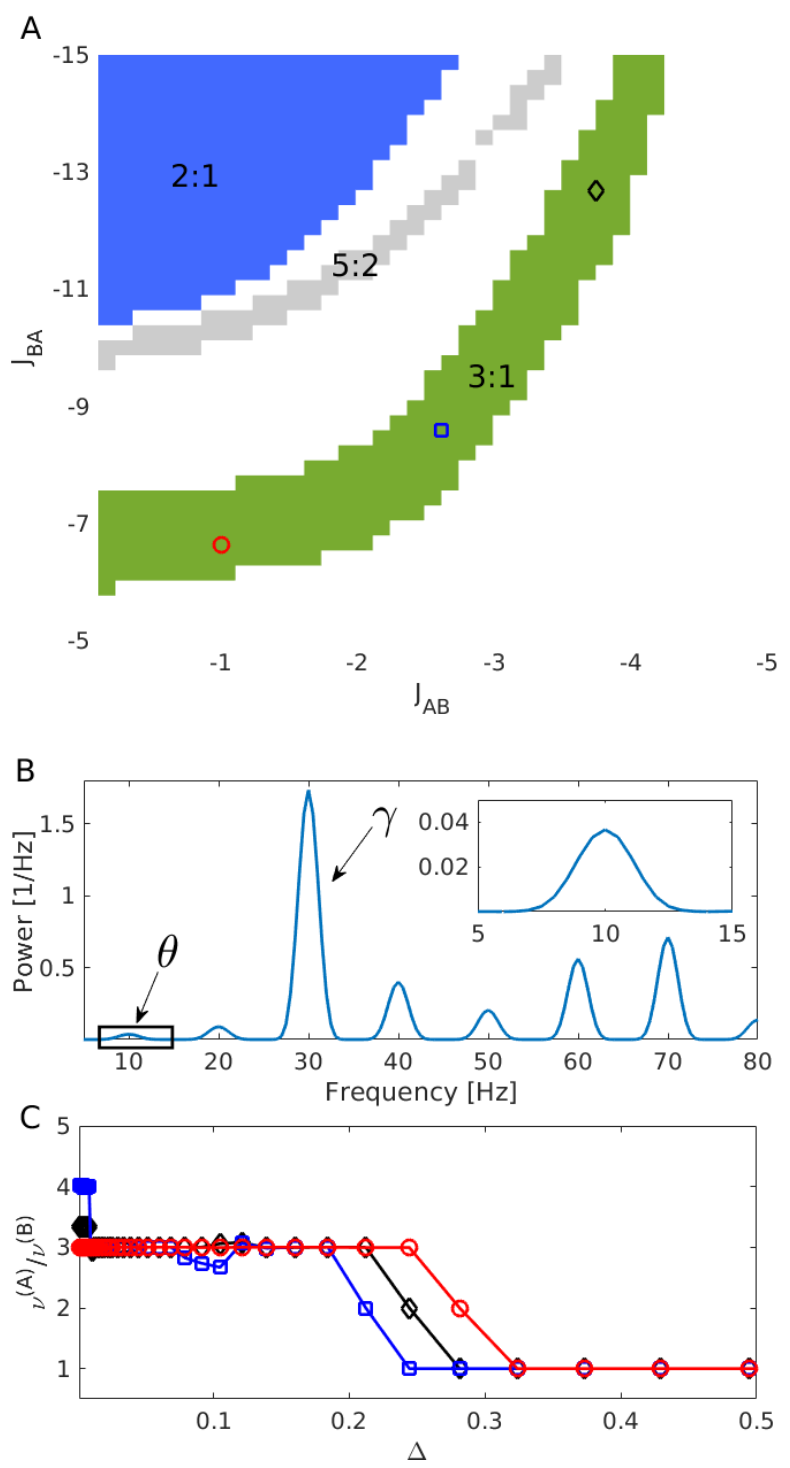

FIG. 9. CFC in bidirectionally coupled populations with an external $\theta$ modulation. A) Locking modes for different values of the cross-coupling. Red circle denotes the pair $\left\{J_{A B}, J_{B A}\right\}$ giving rise to the maximum value of $\rho_{31}$ which is used for simulations in B-C). Black diamond and blue square correspond also to 3:1 modes with smaller order parameter value. B) Power spectrum of the time trace $r^{(A)}(t)$ for the the case denoted by the red circle in panel (A), the inset displays an enlargement corresponding to the $\theta$-band. C) Ratio of the fast and slow population frequencies $\nu^{(A)} / \nu^{(B)}$ showing the extend of the 3:1 mode for the three values of $\left\{J_{A B}, J_{B A}\right\}$ depicted by the symbols in A) at varying values of disorder $\Delta:=\Delta^{(A)}=\Delta^{(B)}$. For the $\theta$-forcing current we set $I_{0}^{(B)}=0.5$ and $\nu_{\theta}=10 \mathrm{~Hz}$, all the other parameters as in Fig. 8 . 
bioRxiv preprint doi: https://doi.org/10.1101/745828; this version posted August 24, 2019. The copyright holder for this preprint (which was not certified by peer review) is the author/funder, who has granted bioRxiv a license to display the preprint in perpetuity. It is made available under aCC-BY-NC-ND 4.0 International license.
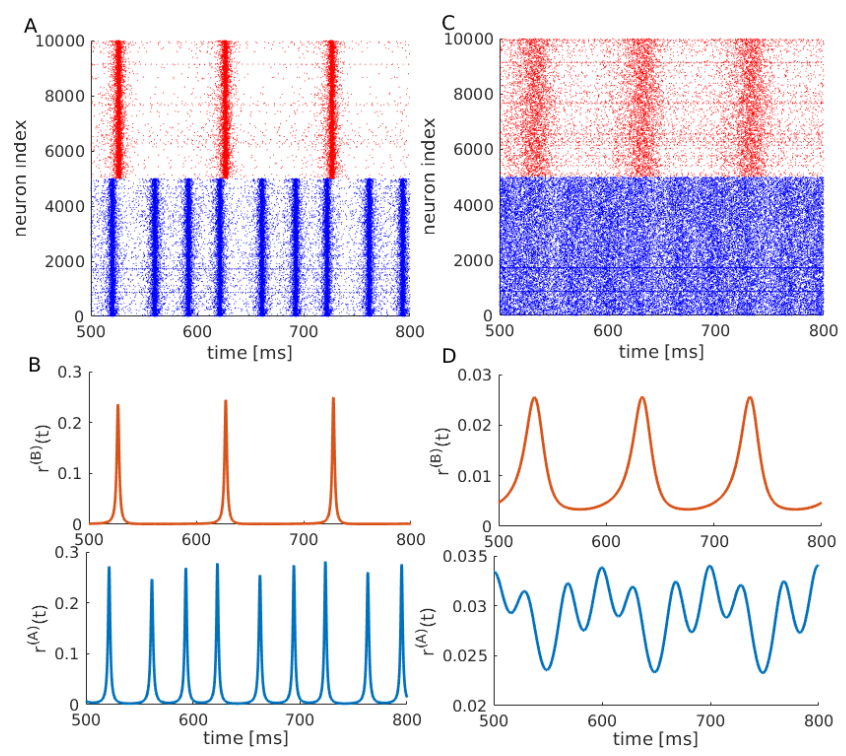

FIG. 10. P-P and P-A couplings in presence of a $\theta$ forcing. A) Raster plot of the network model Eq. (1) showing the fast and slow population in blue and red colors respectively for the same system analyzed in Fig. 9 with the optimal pair $\left\{J_{A B}, J_{B A}\right\}=\{-1,-6.63\}$ and $\Delta=0.05$. B) Time traces of $r(t)$ for the fast (blue) and slow (red) populations for the case depicted in the raster plot in A). C-D) same as in A-B) for the same parameters except for $\Delta=0.2$. Parameters as in Fig. 9. 